\title{
Procesos de gestión pública innovadora: el caso de los módulos de atención a migrantes en Baja California José Ascención Moreno Mena*
}

\section{Resumen}

En esta investigación se presentan dos ejemplos que permiten ver las posibilidades y los obstáculos de las alianzas estratégicas entre la sociedad civil y los gobiernos locales para desarrollar programas que ayuden a solucionar los problemas que la comunidad demanda. Los estudios analizados se desarrollan en el estado de Baja California y se basan en información documental y en entrevistas a informantes claves, en este caso líderes de los programas. El primero de ellos tiene que ver con la experiencia de una oficina de apoyo al migrante en Mexicali, dirigida exclusivamente por una organización civil; mientras que el segundo se refiere a un programa para la atención a población migrante deportada de Estados Unidos, que ha sido utilizado por el gobierno federal como punta de lanza de una política pública más amplia en el ámbito regional. Los dos ejemplos representan casos de gestión pública innovadora. Se presentan los avances y obstáculos para llevar a cabo ese tipo de procesos. Asimismo, se concluye que estos ejemplos pueden ser el germen de una nueva gobernanza.

Palabras clave: Políticas públicas, gestión asociada, organizaciones civiles.

\begin{abstract}
Two examples that allow surmising the possibilities and obstacles of strategic alliances between the civil society and local governments seeking to develop programs that help solve the problems the community demands are featured in this research. The studies that were analyzed take place in the State of Baja California, and are based on documentary information and on interviews with key informants, which in this case, are the program leaders. The first of them has to do with the experience of a migrant support office in Mexicali, which is under the exclusive direction of a civil organization; while the second entails a program for the attention of migrants that were deported from the United States, whereby such program has been used by the federal government to spearhead a more encompassing public policy in the regional sphere. The two examples portray innovative public stewardship cases. The breakthroughs and obstacles to carry out such types of processes are presented. Furthermore, it is concluded that these two examples may be the seed of a new governance.
\end{abstract}

Keywords: Public policies, associated management, civil organizations.

* Investigador del Instituto de Investigaciones Sociales de la Universidad Autónoma de Baja California. Correo electrónico: jmoreno@uabc.mx 


\section{Introducción}

La globalización ha puesto en la agenda de discusión la participación de nuevos actores sociales en los asuntos públicos que claman participación en el diseño y la implementación de políticas públicas. Las organizaciones civiles (OC) ${ }^{1}$ de América Latina han comenzado a explorar esquemas innovadores de gestión pública con los gobiernos locales, lo que ha traído la necesidad de ser explicados con nuevos conceptos de la administración pública como la gestión asociada. ${ }^{2}$

En México, las dificultades para conducir y orientar el desarrollo local a partir de la articulación de recursos económicos y naturales, y de la formación de consensos entre los diversos actores locales, son innumerables. Si bien es cierto que estos procesos pasan por la complejidad de la estructura de gobierno y que no siempre hay correlación con las relaciones intergubernamentales, también lo es que se han presentado algunas condiciones propicias para la participación de la sociedad civil en los asuntos de gobierno, sobre todo en el ámbito local.

Actualmente existen muchos ejemplos de cogestión entre la sociedad civil y el gobierno, sin embargo, éstos se enfrentan a múltiples obstáculos. El objetivo principal de este documento es analizar dos ejemplos de gestión pública innovadora para identificar las posibilidades y dificultades que enfrentan los procesos en su intento por sustituir los esquemas tradicionales de la administración pública. El primer caso se refiere a la creación de un módulo de atención a migrantes repatriados en la garita de Mexicali-Caléxico, mismo que es coordinado por las organizaciones civiles Albergue del Desierto y el Centro de Apoyo al Trabajador Migrante, las cuales funcionan con recursos propios; el segundo caso lo constituye un programa de atención integral a migrantes deportados en Tijuana, el cual ha sido desarrollado por la red de oc Coalición Pro Defensa del Migrante

\footnotetext{
1 Retomamos el concepto de Olvera (2001:37) sobre organizaciones civiles como aquellas "asociaciones libres y voluntarias de ciudadanos cuyo fin es actuar conjuntamente en el espacio público para contribuir a la resolución de los problemas de la sociedad y llenar los vacíos de atención a las necesidades de la población dejados por la acción del Estado y del mercado”.

2 Más adelante, en el primer apartado, se presenta una definición de este concepto.
} 
con apoyo gubernamental. Ambos ejemplos se desarrollan en el estado de Baja California y son resultado de estudios basados fundamentalmente en información documental y en entrevistas a informantes claves, en este caso líderes de los programas.

La investigación sostiene que a pesar de los intentos por desarrollar programas de gestión pública innovadores, éstos tienden a obstaculizarse por la inexistencia de una cultura política y de gestión que incorpore la legalidad, la transparencia, la rendición de cuentas y el sentido social de la acción gubernamental (Ramos y Reyes, 2005). En el sector gubernamental privan todavía las visiones de gestión tradicionales que ven a los ciudadanos como objetos destinatarios de las acciones de gobierno, y cuando una organización civil se decide a aplicar un programa de política pública, es vista con sospecha. Por otro lado, las organizaciones civiles no asumen el potencial que tienen como entes en el desarrollo social por falta de una estructura que les permita la autonomía en los recursos, y se convierten así en meros receptores de ayuda gubernamental, reproduciendo vicios como el paternalismo, el clientelismo y la falta de transparencia. O bien, existe poca voluntad de las organizaciones civiles para desarrollar procesos de gestión asociada por temor a ser cooptadas y subordinadas.

A manera de marco de referencia, el trabajo comienza por explicar las nuevas concepciones de políticas y gestión públicas, así como la participación de las oc en las políticas de desarrollo social. En un segundo apartado se presentan los antecedentes de los programas de atención a migrantes en Baja California, y los dos ejemplos, para concluir con algunas consideraciones y sugerencias.

\section{Nuevos cambios en las concepciones de políticas y gestión públicas}

En los últimos años -sobre todo a partir de los procesos de globalización y la cada vez más importante participación de la sociedad civil en los asuntos públicos- se ha reformulado la vieja concepción de la gestión pública concebida simplemente como un medio para administrar los servicios y productos del Estado, y poco adecuada para los tiempos modernos. Entre las deficiencias más comunes de esta concepción de la gestión pública podemos mencionar las siguientes: 
- Objetivos poco claros y ausencia de evaluación.

- Enfoque hacia adentro, centralizado y con excesivas reglas y normas que impedían la competencia, establecer responsabilidades y la motivación para mejorar.

- Clara falta de técnicas de gestión, que desembocaba en una excesiva función pública burocratizada.

- Una administración enfocada al ciudadano-servidor (Fernández, Fernández y Rodríguez, 2008).

Así, el viejo modelo de gestión pública se vio limitado para responder al nuevo entorno socioeconómico que se produce en los distintos países, y como respuesta surgió en los años ochenta el paradigma de la Nueva Gestión Pública (NGP), que propone una visión más flexible y descentralizadora de la función pública, adoptando los métodos de la gestión del sector privado con miras a obtener resultados en términos de eficiencia, eficacia y calidad del servicio.

A diferencia de los modelos tradicionales, la NGP da especial énfasis al establecimiento de objetivos concretos y a las metas cuantificables y medibles, procurando el ahorro y la reducción de costos; asimismo, incentiva el uso de las nuevas tecnologías y de la competencia, y se centra en la mejora de la cantidad y la calidad de los servicios públicos con el fin de que se favorezca la transparencia y la rendición de cuentas.

Esta nueva visión, orientada hacia el ciudadano, considerado usuariocliente, da a éste un peso específico en la toma de decisiones y en las acciones del organismo público, sobre todo en lo referente a la distribución de los recursos y a la provisión de los servicios; es decir, pretende propiciar procesos de feedback donde el usuario-cliente esté más cerca de la toma de decisiones y pueda reaccionar ante la gestión desarrollada de tal manera que se lleve a cabo una retroalimentación (Fernández, Fernández y Rodríguez, 2008).

Si bien el enfoque de la NGP no está exento de fundadas críticas en su aplicabilidad, sobre todo por las disparidades políticas que obstaculizan su implementación en algunos países, como sostienen Fernández, Fernández y Rodríguez (2008), su establecimiento ha traído cambios notables en la forma de actuación de las distintas administraciones públicas, sobre todo en el aspecto de la transparencia y en la flexibilidad en su gestión, además de que, lo más importante, se ha abierto más a los ciudadanos. 
En ese contexto de cambios, la concepción de las políticas públicas también ha sufrido una transformación, acorde con las nuevas realidades que se presentan en el espacio público, especialmente la relacionada con la creciente participación ciudadana. Autores como Gómez y Moctezuma (1999) advierten que las políticas públicas no deben confundirse con las políticas de gobierno, ya que estas últimas se orientan hacia la conservación del Estado como ente orgánico, con vida propia, a través de sus instituciones, y se rigen por principios legales, centrándose en resolver los problemas más urgentes con visiones de corto y mediano plazos. Desde esta perspectiva, las políticas de gobierno se rigen de acuerdo con la legislación vigente, y los gobiernos federal y locales deben inscribir sus acciones a partir del marco que les da origen y los regula.

En cambio, las políticas públicas en su concepción moderna, como lo sostienen los autores mencionados,

[...] no resultan de la gran decisión instantánea en la cúspide del gobierno, sino de un proceso en el que se entremezclan las iniciativas de las organizaciones sociales y de las instancias gubernamentales, abordando las etapas de elaboración de las políticas, agendando o decidiendo las cuestiones o demandas de interés público y ponderando acciones para encararlos (Gómez y Moctezuma, 1999:24).

Sobre el mismo aspecto, Cabrero (2003) argumenta que el análisis actual de las políticas públicas asume la esfera de lo público como el espacio de confluencia entre actores gubernamentales y no gubernamentales, lo cual implica un cierto grado de pluralidad en la acción pública porque ya no es un solo actor el que toma las decisiones, sino que éstas se presentan en corresponsabilidad. Sobre el mismo aspecto, Canto (2002) ofrece una definición en la que se consideran también el contexto y el medio ambiente, espacios donde se desarrollan las políticas y las relaciones de poder entre los distintos actores:

Las políticas públicas son cursos de acción, tendientes a la solución de problemas públicos, definidos a partir de la interacción de diversos sujetos sociales y de relaciones de poder, que pretenden utilizar de manera más eficiente los recursos públicos y tomar decisiones a través de mecanismos democráticos, con la participación de la sociedad (Canto, 2002:70). 
Estas visiones, basadas en los nuevos escenarios de los países democráticos y en los cambios en el papel de los Estados, tratan de adecuarse a los nuevos procesos asociativos y a la redefinición de las relaciones que establecen los distintos actores con el poder, en donde el monopolio estatal de lo público se pone en cuestionamiento. En el medio académico surge entonces el concepto de lo público-no estatal para explicar las acciones colectivas de los actores no gubernamentales que desde lo privado intentan incidir en las políticas públicas (Reyes, 2008).

Organismos multilaterales como el Banco Mundial (вм), el Banco Interamericano de Desarrollo (BID) y el Programa de las Naciones Unidas para el Desarrollo (PNUD) han advertido estos procesos y, por eso, en sus recomendaciones para transitar hacia la democracia sugieren a los gobiernos hacer uso del concepto gobernance o gobernancia que, según su concepción, implica habilitar y fortalecer la participación y el control ciudadano en su diversidad de formas a efecto de encontrar equilibrios complejos que no reduzcan el desarrollo a un único actor, sea éste el Estado o bien el mercado. Desde esta perspectiva, una situación ideal de gobernance implica que ésta debería estar orientada al consenso, ser participativa, equitativa e inclusiva, responsable, abierta a la rendición de cuentas, transparente, efectiva y eficiente, además de asegurar el Estado de derecho (Wagner, 2008).

Desde un punto de vista complementario, la corriente intergubernamentalista, referenciada por Vargas (2005), nos dice que

[...] las conexiones para mejorar la administración y construir una gobernabilidad efectiva son el desempeño administrativo del gobierno, los ciudadanos que se incluyen en la medición del desempeño, los ciudadanos que se involucran en las políticas gubernamentales y en su implementación y, finalmente, el alineamiento estratégico de los tres elementos de gobernance $[\ldots]$

Estas concepciones son definidas obviamente en el contexto de un país democrático, con esferas públicas bien definidas donde las reglas del juego son claras y existe una vocación democrática de los distintos actores políticos (lo que no los exenta de tener conflictos), que implica la existencia de canales de información eficientes entre la sociedad civil y el Estado; más aún, la existencia de posibles consensos y espacios donde se contemplan 
marcos jurídicos apropiados que permiten un peso específico de los distintos sectores en las decisiones políticas.

En países como el nuestro, a pesar de la alternancia en el gobierno que ya se da en algunos estados -como Baja California-, debemos advertir que no siempre existe un sistema político formalmente conformado que facilite la participación de las organizaciones civiles en las diversas instancias de toma de decisiones políticas. Ni tampoco existe la cultura democrática de los gobernantes que permita una visión de colaboración mutua sin subordinación entre sociedad civil y gobierno. Por el contrario, persiste un bajo nivel de institucionalización y deficientes canales de acceso al sistema político, asimismo prácticas autoritarias reconocidas (tanto por el Estado como por la sociedad civil) que impiden llevar adelante procesos de políticas públicas en su concepción moderna, donde tampoco las organizaciones civiles han procesado su potencial de entidades innovadoras.

Sin embargo, es justo reconocer que en la actualidad hay una tendencia que mira hacia la construcción de mejores condiciones para la participación de la sociedad civil en las políticas públicas, sobre todo en el ámbito municipal. Muchas de las decisiones políticas o económicas más importantes, otras consideradas patrimonio exclusivo del gobierno, hoy pasan por el tamiz de la discusión entre los diversos actores sociales, que reclaman, proponen e incluso elaboran propuestas alternativas a las del gobierno, reivindicando su derecho a participar no sólo en la consulta, sino también en los procesos de decisión.

Otro de los conceptos que se han acuñado paulatinamente para explicar los procesos de corresponsabilidad entre la sociedad civil y el gobierno es el de gestión asociada, el cual refiere a una alianza estratégica entre las oc y el Estado que permite optimizar recursos y ampliar la capacidad de respuesta a las demandas sociales; además de obligar a los actores a diversificar los servicios y crear la necesidad de ganar en transparencia y legitimidad. Clemente (2000) define la gestión asociada como

[...] una alianza de trabajo entre diferentes actores sociales, cuyo interés común es el desarrollo social y económico en cualquiera de sus dimensiones y escalas. Se trata de un modelo donde las partes, a partir de acuerdos explícitos y públicos, se asocian para resolver problemas de interés social (Clemente, 2000:118). 
Este autor también menciona una serie de aspectos indispensables para llevar a cabo la gestión asociada: en principio, debe existir una relativa autonomía de cada uno de los componentes de la alianza, lo que permitirá cierto margen de libertad de los participantes; segundo, los actores deben tener representatividad y gozar de legitimidad; tercero, la democracia en los vínculos de los participantes debe estar presente; y un agregado de Poggiese (1993), tener la voluntad de configurar una experiencia democratizante que, en mérito a su efectividad, devuelva la credibilidad y confiabilidad a la democracia como sistema de conjugación de intereses y debate sobre el destino de la sociedad; y por último, debe existir una contribución diferenciada y complementaria de las partes. Asimismo, en un escenario de gestión asociada Poggiese (1993) indica que el Estado deberá tener la voluntad política para buscar la cogestión, no sólo enunciativa sino con verdadero compromiso; tener la sensibilidad para dar y recibir confianza, en el plano de los procesos decisorios; buscar la cooperación de manera horizontal y no el simple relacionamiento, donde los que tienen la capacidad de decisión pretendan utilizar a las oc para sus intereses políticos; finalmente, el Estado debe contribuir al fortalecimiento y desarrollo de las organizaciones civiles para que estén en condiciones de co-participar.

Ramos y Reyes (2005) consideran además que debe existir en ambas partes una apertura hacia la cultura y la política de gestión alejada de la visión tradicional, la cual está plagada de corrupción y es clientelar, paternalista y de subordinación; sin esta nueva visión no será posible una gestión asociada efectiva para el desarrollo social. ${ }^{3}$ En otras palabras, es necesario un enfoque donde se promueva que el público ciudadano deje de ser un objeto y destinatario de las acciones de gobierno para convertirse en un sujeto activo, partícipe en el diseño, la implementación y la solución de los problemas sociales.

Otro elemento importante para promover la gestión asociada es el fortalecimiento de las capacidades del gobierno para incentivar la gobernación, y el fortalecimiento institucional de las organizaciones civiles que permita incidir en la agenda pública (Ramos y Reyes, 2005).

3 Los valores que acompañan a la cultura y la política de gestión son la legalidad, la transparencia y la rendición de cuentas (Ramos y Reyes, 2005). 
En resumen, se están presentando nuevas realidades en las relaciones de poder que buscan satisfacer las necesidades que la sociedad demanda pero éstas se presentan en los Estados democráticos y en escenarios ideales; sin embargo, estos elementos nos pueden servir para comparar la relación entre las organizaciones civiles y el gobierno en escenarios distintos, como el de nuestro país. De ahí la importancia de analizar algunos casos que se han presentado en el ámbito local.

\section{Hacia la búsqueda de corresponsabilidad entre la sociedad civil y el gobierno}

Ante el crecimiento de la participación ciudadana, a través de las oc, en los asuntos del desarrollo social, los gobiernos de los distintos países han implementado de manera progresiva nuevas visiones de administración y gestión públicas, si bien a veces con algunas dificultades o distorsiones. Estas dificultades se incrementan cuando no se tienen el marco propicio ni la voluntad política para cambiar esquemas caducos y burocráticos, y se reflejan más en países como México que apenas está experimentando una incipiente transición hacia la democracia.

En America Latina, aun con estas limitaciones, las oc han comenzado a explorar esquemas innovadores de gestión con los gobiernos locales en distintos países, esto con la intención no sólo de ser receptoras de los servicios públicos sino también promotoras, organizadoras e incluso fiscalizadoras de los mismos, lo cual no implica la renuncia o el desconocimiento de la responsabilidad de las instituciones gubernamentales sino, por el contrario, incorpora el involucramiento de la ciudadanía en la atención de sus propios problemas.

En México estos intentos se han dado sobre todo en el ámbito local, por las posibilidades que esto genera. Al respecto, Cabrero (2003) sostiene que es precisamente en los gobiernos locales donde se amplía la visión y el espacio de intervención, expandiendo y profundizando las agendas tradicionales de políticas públicas hacia una agenda más integral, como las políticas amplias de participación ciudadana, protección ambiental, desarrollo económico, bienestar social, educación y salud.

Estos procesos, siguiendo a este autor, obligan a construir redes de actores en torno a la acción pública y, al mismo tiempo, a innovar en materia 
de gestión. Es ahí donde pueden surgir nuevas formas de concertación entre la sociedad y el Estado, tipos de construcciones político-técnica-comunitarias con un sentido de poder compartido que se contrapongan a la tradicional práctica autoritaria de nuestros gobiernos.

Sin embargo, y a pesar de los obstáculos, existen experiencias creativas de desarrollo social que contribuyen a elevar la calidad de vida de la población en los gobiernos locales, que bien pueden ser consideradas como intentos de modelos de gestión pública asociada donde algunas resultan exitosas y perduran, mientras que otras se truncan por diversos factores. ${ }^{4}$

Poco a poco se han creado los marcos normativos que propician la participación de la sociedad civil organizada en los asuntos públicos, como por ejemplo la Ley de Fomento para las Organizaciones Civiles, federal y estatales, así como las leyes locales de desarrollo social que incorporan la participación ciudadana, las cuales pueden ser el vehículo que contribuya a acelerar la creación de gestiones innovadoras.

Actualmente existen múltiples ejemplos de procesos de co-gestión entre la sociedad civil y el gobierno, los cuales se expresan en convenios, contratos u otras formas. A continuación se presentan dos ejemplos que expresan las dificultades y los avances de los intentos por crear gestiones innovadoras.

\section{Programas de atención a la población migrante en Baja California: antecedentes}

Desde principios de los años noventa las organizaciones civiles de apoyo a la población migrante en Baja California habían demandado la intervención del gobierno estatal para atender a los migrantes deportados de Estados Unidos por las ciudades de la entidad, para ello diseñaron líneas generales y acciones específicas para desarrollar un posible programa de atención integral alejado de los modelos tradicionales eminentemente

\footnotetext{
${ }_{4} \mathrm{Al}$ respecto, el CIDE, El Colegio de la Frontera Norte (El Colef) y la Secretaría de Gobernación (Segob) han establecido concursos para premiar los mejores proyectos de gestión públicas innovadora en el país; algunos han sido publicados en forma de libros. Véase Guillén, González y Rojo (2007).
} 
asistenciales, pero como el problema no era considerado prioritario por el gobierno local, no se obtuvo una respuesta positiva, aun así, se insistió en ponerlo en la agenda de discusión.

A partir de 1995, con la puesta en marcha de políticas de control migratorio por parte de las autoridades estadounidenses, la demanda de las oc se convirtió en una necesidad. La construcción y el reforzamiento de una triple barda en la frontera San Isidro-Tijuana selló prácticamente esa zona de cruce de migrantes y la deportación masiva de indocumentados por la garita Mexicali-Caléxico provocó una coyuntura favorable para volver a proponer el programa integral.

La necesidad de difundir y crear sinergias para incidir en la agenda gubernamental obligó a las oc a articularse en redes; de esta forma, en Mexicali se conformó el Frente Amplio de Apoyo al Migrante (Frapam) [Avendaño, Moreno y Priego, 2000], que aglutinó a grupos que desarrollaban acciones en pro de los migrantes como también a organizaciones gremiales y sociales del movimiento urbano popular, e incluso a la Subprocuraduría de los Derechos Humanos y Protección Ciudadana de Baja California, que era una institución gubernamental.

Blanca Villaseñor, una de las protagonistas del Frente, en una entrevista a la revista local Paralelo 28 relata lo anterior:

El trabajo entre las diferentes organizaciones tiene su origen en la actividad del Frente Amplio de Apoyo al Migrante, cuando hicimos algunas acciones urgentes al iniciarse la operación Guardián hace 5 años. Esto hizo que salieran organizaciones ligadas directamente al trabajo de la problemática migratoria, de defensa de los derechos humanos y en otras aéreas de trabajo (Sánchez, 2000).

En ese tiempo las deportaciones se recrudecieron en Mexicali de tal forma que se llegaron a reportar más de 500 por día, y con ellas se incrementaban también las violaciones a los derechos humanos. La política del gobierno estadounidense era inhibir el cruce por las zonas tradicionales obligando a los migrantes a atravesar por zonas inhóspitas, al mismo tiempo, deportar a los connacionales por lugares lejanos a donde se internaban hacia Estados Unidos. Ciudades como Mexicali nunca habían experimentado esa magnitud de población deportada. 
En ese contexto, el Frapam inició la lucha por que se adoptara un programa temporal de emergencia de atención integral a los migrantes repatriados, el cual contemplara transporte hacia los albergues, salud, asesoría jurídica, retorno a sus lugares de origen y bolsa de trabajo en Tijuana y Mexicali.

Se pretendía que el gobierno estatal interviniera en el auxilio a esta población vulnerable, y para ello, el Frapam utilizó como mecanismos de presión conferencias de prensa y la difusión masiva de algunos resultados de monitoreos efectuados en la garita internacional de Baja California, en los que se mostraban datos sobre violaciones a los derechos humanos de los migrantes repatriados y las paupérrimas condiciones en que venían del vecino país. Estas acciones, inéditas hasta entonces en la región, buscaban el apoyo popular para hacer "visible" el problema y elevarlo a la agenda gubernamental con el fin de propiciar la intervención de las autoridades gubernamentales estatales, que se negaban a intervenir por considerar que era un problema que le competía al gobierno federal.

La capacidad del Frapam para articular y poner en acción a diversas oc, logrando una alianza coyuntural por una demanda concreta con objetivos bien definidos, le confirió legitimidad y conformó un movimiento social estructurado que permitió incidir coyunturalmente para que el gobierno interviniera en la atención a los migrantes -lo cual se logró parcialmente, al establecer una alianza estratégica con el gobierno estatal que creó un programa interinstitucional de apoyo al migrante deportado que duró algunos meses, hasta que se dio un cambio de gobierno-. Las organizaciones civiles fueron el puntal de ese programa.

De esa manera, las organizaciones civiles aglutinadas en el Frapam tuvieron la capacidad de elevar a políticas públicas un problema social sentido en las ciudades fronterizas de Baja California. Las oc también presentaron propuestas alternativas y lograron participar en la aplicación de las políticas públicas, pero no llegaron al proceso de evaluación y seguimiento ni lograron formalizar la red para darle continuidad. Por parte del nuevo gobierno, no existió una visión estratégica ni voluntad política que permitiera continuar con el proyecto.

Así, la falta de continuidad de la red, aunada a una situación política desfavorable, truncó el proceso; aunque hay que reconocer que la estructura orgánica de un frente coyuntural no garantizaba poder sostener un 
programa de largo plazo. En este caso fueron factores organizativos internos y externos los que impidieron que el programa continuara. ${ }^{5}$

Sin embargo, esto fue el antecedente para la creación de la Oficina de Atención al Migrante en Mexicali y el surgimiento de la Coalición Pro Defensa del Migrante de Baja California.

\section{La Oficina de Atención al Migrante del Albergue del Desierto en Mexicali ${ }^{6}$}

Con la intensificación de las acciones de la Operación Guardián en 1995, y los efectos que trajo -cambio de rutas migratorias, muertes de migrantes y repatriaciones por lugares alejados de las zonas tradicionales de cruce-, las organizaciones civiles de Mexicali se instalaron directamente en los lugares donde se repatriaba a los migrantes para brindarles una mejor atención; así lo relata una de las líderes del Albergue del Desierto:

[...] debíamos tener presencia con el migrante deportado, atenderlo aunque fuera de una forma rudimentaria, otorgando comida y alguna información que lo orientara porque llegaba a una ciudad que desconocía. Al entonces gobernador Terán, en el marco del programa de interinstitucional de apoyo a la población deportada, donde el Frente Amplio de Apoyo al Migrante participaba, le solicitamos un techadito para atender a los migrantes, el cual nos facilitó. Posteriormente integramos algunas bancas y con eso iniciamos

\footnotetext{
5 Entre los factores organizativos se tiene que había distintos liderazgos dentro de la red, algunos con intereses a largo plazo, y no existía un estatuto o reglas básicas que permitieran una continuidad, además de que algunos de los actores no tenían el respaldo de una organización y se integraban de manera individual; entre los factores externos, el que mayor peso tuvo fue la muerte del entonces gobernador del estado, Héctor Terán, y la llegada de un nuevo gobernante que desconoció los acuerdos establecidos con el anterior gobierno.

6 El Albergue del Desierto es una organización civil conformada hace 20 años en la ciudad de Mexicali; su objetivo principal es atender a menores migrantes, sin embargo, desde 1995, con el recrudecimiento de las medidas de control migratorio en Estados Unidos, amplió sus servicios a mujeres migrantes -el nombre que utilizaba anteriormente era Albergue Juvenil del Desierto-. La organización civil cuenta con un albergue para menores ubicado a varios kilómetros de la garita internacional.
} 
la atención en la garita. En 1995 las autoridades de aduana nos avisaron que quitarían el techado porque pensaban construir algunas oficinas en ese espacio. Entonces comenzamos una larga lucha por adquirir uno de esos locales o módulos (entrevista, Mónica Oropeza, 2009).

La lucha de las oc por conseguir un espacio para atender directamente a los migrantes repatriados ${ }^{7}$ en las inmediaciones de la garita internacional se volvió ardua y llena de tropiezos ya que las autoridades, en este caso federales, no concebían que una organización civil defensora de migrantes estuviera en terrenos del gobierno, mucho menos que participara en la atención de los migrantes deportados; sin embargo, sus acciones le daban legitimación y aseguraban su derecho a atender a esta población:

Había mucha resistencia por parte de las autoridades gubernamentales para otorgar una oficina a una ONG, eso no estaba contemplado en sus planes. Pero ya teníamos el reconocimiento de los funcionarios del Instituto Nacional de Migración y de aduanas, porque fuimos los primeros que estuvimos en la línea. Los mismos funcionarios nos canalizaban a los migrantes deportados que requerían algún servicio. Nos ganamos su confianza porque íbamos a ayudar a los migrantes (entrevista, Mónica Oropeza, 2009).

Gracias a una coyuntura política favorable en el país, donde, por un lado, se tenía el apoyo del gobierno estatal a la organización civil de Baja California y, por otro, se propiciaba una política de acercamiento del entonces presidente de la República Ernesto Zedillo Ponce de León con las oc en general, fue posible lograr romper las barreras burocráticas y otorgar la concesión de un espacio en terrenos federales a una organización civil; así lo relata una de las protagonistas:

Nos entrevistamos con muchos funcionarios del gobierno federal y después de un año de tanto caminar, mandando cartas, nos dirigimos con el ingeniero Esquivias, delegado de la Comisión de Avalúos de Bienes Nacionales (CABIN)

\footnotetext{
7 Aunque los conceptos repatriado y deportado tienen connotaciones distintas por el carácter jurídico del hecho, en este trabajo los utilizamos como sinónimos porque la atención no distingue quiénes entran en cada una de las categorías.
} 
aquí en Mexicali, y con el apoyo de Federico García Estrada, subprocurador de la Procuraduría de los Derechos Humanos y Protección Ciudadana de Baja California, y el delegado del Instituto Nacional de Migración que avalaban nuestro trabajo, se logró la promesa del ingeniero que trataría de intervenir; sin embargo, él mismo nos anticipó que resultaría muy difícil porque no existía la practica de facilitar a una ONG un local del gobierno. Finalmente, en 1996, después de tanto insistir, se nos otorgó un local en comodato, pagando un arrendamiento (entrevista, Mónica Oropeza, 2009).

El resultado favorable de las gestiones de la oc fue posible gracias al apoyo de otros actores gubernamentales, lo que terminó por disipar las dudas que se tenían sobre la participación de una organización civil en programas públicos. Desde nuestro punto de vista, se sentó un precedente importante de gestión innovadora en la localidad, pues por primera vez una organización civil privada (Albergue del Desierto) desarrollaba con sus propios recursos acciones públicas en una oficina ubicada en terrenos gubernamentales, concesionada mediante arrendamiento por el propio gobierno federal.

La oc responsable integró de manera permanente a otra organización civil, el Centro de Apoyo al Trabajador Migrante, ${ }^{8}$ y en forma temporal a voluntarios de la Iglesia católica, así como a Médicos del Mundo, organización de carácter internacional. De esta manera se conformó una oficina que dio cobijo a varias iniciativas que favorecerían a los migrantes repatriados por la garita Mexicali-Caléxico. ${ }^{9}$

La oficina funciona de manera autónoma al gobierno en materia de recursos, pero canaliza a los migrantes repatriados según sus necesidades hacia las instituciones gubernamentales o bien a los distintos albergues de la ciudad. A pesar de recibir voluntarios extranjeros de la Iglesia católica, las oc responsables de la oficina mantienen su carácter laico.

En ese espacio se recibe principalmente a la población repatriada proveniente de San Diego, Los Ángeles y los centros de detención del Valle

8 El Centro de Apoyo al Trabajador Migrante es una organización civil cuyo objetivo es brindar atención a los migrantes adultos. Comparte recursos humanos con el Albergue del Desierto.

9 Los responsables de la oficina le denominan "Módulo de Atención al Migrante”. 
Imperial; se otorga asistencia social mediante refrigerios, orientación y asesoría, según sus necesidades; también se obsequia ropa y calzado; se establece comunicación con sus familiares y se canaliza a las personas que llegan con algún problema de salud a las instituciones pertinentes; se lleva a cabo recopilación de información para fundamentar denuncias o bien documentar el fenómeno migratorio en la localidad, lo que se realiza mediante boletines de prensa, folletos, libros, etcétera. ${ }^{10}$

Las oc responsables de la oficina mantienen una buena relación y tienen convenios con instituciones gubernamentales como la Procuraduría de los Derechos Humanos y Protección Ciudadana de Baja California, la delegación de la Comisión Nacional de los Derechos Humanos (CNDH), el DIF estatal y municipal, las universidades del estado que aportan prestadores de servicios sociales, etcétera.

Aunque la oficina funciona en mayor medida con recursos propios, esto no invalida que las oc reciban apoyos gubernamentales mediante los fondos de conversión a los que convoca la Secretaría de Desarrollo Social (Sedesol) cada año. ${ }^{11}$

El modulo depende del Albergue en el cien por ciento. Nunca hemos recibido una propuesta para financiar la oficina en forma directa. Siempre hemos cuidado nuestra independencia, porque lo contrario nos supedita a los caprichos de los funcionarios (entrevista, Mónica Oropeza, 2009).

A más de 10 años de su funcionamiento, los intentos por mantener su autonomía han traído a las oc una serie de fricciones con las autoridades gubernamentales de carácter federal, especialmente cuando se han desarrollado acciones de denuncia y documentación de violaciones a los derechos humanos; eso lo relata Mónica Oropeza, responsable de la oficina:

\footnotetext{
$10 \mathrm{Al}$ respecto, las organizaciones responsables de la oficina ya han publicado dos libros.

11 Los fondos de conversión son recursos que pone a concurso la federación por medio de la Sedesol, la Secretaría de Desarrollo del Gobierno de Baja California y la Secretaría de Desarrollo Municipal. Las organizaciones civiles que cubren los requisitos solicitados participan con un porcentaje del monto asignado al proyecto propuesto, el resto lo otorgan la autoridades.
} 
El tratar de mantener nuestra autonomía nos ha traído fricciones con las autoridades gubernamentales. Algunas han querido regañarnos como si fuéramos sus empleados. Eso nos pasó con un cónsul de México en Caléxico que nos invitó a dialogar a Blanca Villaseñor y mí a su oficina y ahí nos quiso regañar [...] A otros no les gusta que alguien alce la voz y denuncie lo que sucede. En una ocasión vinieron agentes del INAMI a tratar de detener a unos de nuestros voluntarios de nacionalidad alemana, como una represalia de un subdelegado que no le gustó nuestro trabajo. Afortunadamente existía una buena relación con sus jefes en las oficinas centrales de la ciudad de México, lo que impidió que las cosas fueran más allá; luego de algunas reuniones de nuestra directora, Blanca Villaseñor, con los funcionarios se limó la aspereza. Y esto ha sido una constante; en agosto del 2008, el cónsul mexicano en Caléxico nos citó para decirnos que estaba muy enojado porque estábamos violentando la ley, al solicitar la intervención de la Procuraduría de los Derechos Humanos (PDH), ya que según ellos, el Consulado es el que debería intervenir. Nosotros les dijimos que efectivamente son ellos los obligados a levantar las quejas sobre violaciones a los derechos humanos, pero como no lo hacen, entonces nosotros acudimos a otras instituciones. Los migrantes nos presentan sus quejas y nosotros las canalizamos a la PDH, ya que ellos tienen una persona en la garita cerca de nuestra oficina (entrevista, Mónica Oropeza, 2009).

Nuevamente, la visión de subordinación está presente en el enfoque de gestión. Los funcionarios federales ven a las organizaciones civiles como un apéndice del gobierno que les tiene que rendir cuentas a ellos y cuando no lo hacen, obstaculizan su trabajo. No se rigen por el valor público de resolver las necesidades de los migrantes sino por la tradicional visión del control autoritario. Al no existir una cultura de gestión y los marcos normativos suficientes para salvar las controversias, nuevamente se recurre a los canales informales como "las buenas relaciones" con funcionarios de alto nivel.

Los intentos por desaparecer la Oficina de Atención a Migrantes en la garita han continuado a través de los años; otras instituciones gubernamentales han manifestado su inconformidad cuando son señaladas por su ineficacia e indolencia y han solicitado las oficinas de las oc para desarrollar acciones que les competen: 
Por las denuncias que hicimos nosotros por las violaciones a los derechos humanos de menores migrantes por parte de las autoridades estadounidenses y mexicanas, llegaron los del Dif a instalar un módulo, pero ellos querían la oficina que nosotros teníamos ya que argumentaban que nosotros no éramos autoridad de gobierno. Lo bueno es que ya existía un contrato de arrendamiento: Blanca, nuestra directora, tuvo la visión de firmar un documento previo al cambio de gobierno que perduraba hasta el otro periodo, para que no nos movieran fácilmente (entrevista, Mónica Oropeza, 2009).

Afortunadamente, la presencia de años, el papel desempeñado y el prestigio logrado en la comunidad por parte de las organizaciones civiles responsables evitaron las turbulencias políticas y permitieron que el programa continuara operando.

En resumen, a pesar de todas las presiones que ha tenido la Oficina de Atención a Migrantes en Mexicali, se puede asegurar que es un proyecto consolidado, gracias a la estructura de financiamiento que tienen las oc, que no depende del sector gubernamental. Aunque el enfoque de atención es asistencial, sí resuelve las necesidades inmediatas de esa población vulnerable, lo que es reconocido por los actores gubernamentales relacionados con la migración.

El programa de la oficina se inscribe en el enfoque de gestión innovadora, donde la sociedad civil desarrolla políticas públicas destinadas a la población vulnerable. Desafortunadamente, tanto los gobiernos federal como local, y las propias organizaciones civiles, han desperdiciado la oportunidad de establecer una gestión asociada con miras a resolver el problema a largo plazo, como lo proponen Ramos y Reyes (2005); es decir,

[...] una alianza estratégica entre organizaciones civiles y gobierno que permita generar beneficios e impactos sociales en términos de bienestar y calidad de vida y generar beneficios e impactos mediante la adopción de un enfoque proactivo y sustentado en los consensos con distintos actores (Ramos y Reyes, 2005:40).

Los módulos de atención al migrante en Tijuana: una forma de corresponsabilidad entre la sociedad civil y el gobierno

Con el antecedente anterior del Frapam, las organizaciones civiles que atienden a migrantes insistieron en desarrollar un programa de atención 
integral, pero ahora con una estructura más sólida, bajo la coordinación de la Coalición Pro Defensa del Migrante (Coalipro), ${ }^{12}$ red estatal de organismos que atienden y defienden los derechos del migrante y que está conformada por la Casa del Migrante Scalabrini, el Centro Madre Assunta y la Casa ymca, en Tijuana, y por el Albergue del Desierto, el Centro de Derechos Humanos y Educación Cívica y el Centro de Apoyo al Trabajador Migrante, estas últimas de la ciudad de Mexicali; sin embargo, hubo que esperar mejores condiciones políticas en el estado para echar a andar este proyecto.

La oportunidad se presentó con la llegada a la Sedesol del gobierno del estado de un funcionario más sensible a estas problemáticas, que convocó a todos los grupos que atendían a los migrantes a desarrollar un nuevo proyecto en el año 2004. Este nuevo funcionario se comprometió a retomar las propuestas de la Coalipro pero bajo el esquema de una nueva gestión pública en donde se fomentara la corresponsabilidad entre sociedad civil y gobierno. Con esta visión, las organizaciones civiles se encargarían de aplicar las políticas públicas y el estado de apoyar la búsqueda de recursos para la operacionalización así como su seguimiento.

Con estas consideraciones, las oc, encabezadas por la Coalición Pro Defensa del Migrante, partieron de la experiencia anterior del Frapam y diseñaron nuevas estrategias para implementar políticas públicas con una visión de corto y mediano plazos. Así, se partió de la necesidad de crear un programa integral de atención al migrante que no sólo contemplara la asistencia, sino también la investigación, la difusión, la defensa de los derechos humanos, la prevención y otros aspectos relacionados.

De esta manera se impulsó un modelo de trabajo en corresponsabilidad entre el sector gobierno y la sociedad civil. Asimismo, surgió la iniciativa de crear el Comité Estatal de Apoyo al Migrante (Comité Estatal) que conformarían organizaciones civiles defensoras de los migrantes e instituciones públicas relacionadas con el problema migratorio con una estructura flexible formada por comisiones y con un esquema de toma de decisiones bastante horizontal, donde la coordinación recayera en un miembro de las organizaciones civiles (en este caso de la Coalipro). La vo-

12 La Coalición Pro Defensa del Migrante A.C. surgió en 1996 luego de la experiencia del Frente Amplio de Apoyo al Migrante. Esta red tiene un carácter estatal. 
luntad de apertura del gobierno hacia la participación de la sociedad civil en políticas públicas se puso a prueba con este proyecto.

Uno de los primeros resultados de este programa fue la creación de dos módulos de atención a migrantes que iniciaron labores a partir del 23 de febrero de 2005 con el objetivo general de "atender las necesidades de la población migrante deportada por el estado de Baja California de manera integral mediante la recepción, orientación, asistencia y canalización inmediata" (Revista Migrantes, 2005). También se logró convencer a la Sedesol de la necesidad de establecer un programa de coinversión, exclusivamente, para las organizaciones civiles involucradas en el problema migratorio en el ámbito estatal, el cual permitió a algunas de las organizaciones participantes en el modelo tener acceso a recursos económicos.

Para iniciar con el proyecto de los módulos se construyeron dos oficinas en las inmediaciones de las garitas de la Puerta México y Otay en Tijuana. El costo de la construcción física de la infraestructura corrió a cargo del gobierno estatal. El espacio fue proporcionado por la Comisión de Avalúos de Bienes Nacionales (CABIN) y los permisos correspondientes gestionados por el Instituto Nacional de Migración (INM); respecto al personal de atención, éste correspondió a la Coalipro.

Nuevamente, con el cambio de funcionarios, producto del proceso electoral federal del año 2006, se comenzó a entorpecer la actividad del Comité Estatal. Con los cambios en las delegaciones federales de la Sedesol llegaron otros funcionarios públicos con una visión distinta y comenzaron a eliminar los fondos de conversión destinados hacia los migrantes. Eso resultó un duro golpe a las organizaciones civiles de apoyo a esta población.

En el ámbito estatal, si bien se continuó apoyando la labor de la Coalición, el financiamiento para sostener los módulos fue disminuyendo hasta que se presentó la coyuntura electoral estatal del año 2007, donde los actores políticos gubernamentales se olvidaron momentáneamente de los apoyos a las organizaciones y se vieron tentados a tratar de corporativizarlas, cosa que éstas no permitieron.

El Comité dejó de funcionar de manera regular porque las demás organizaciones se fueron retirando paulatinamente al no ver ningún apoyo hacia sus actividades -el programa de los módulos continuó la responsabilidad de la Coalición-. Los miembros de la Coalición iniciaron la búsqueda 
de nuevos recursos con la esperanza de que el gobierno del estado entendiera la necesidad de incorporar el enfoque de gestión pública asociada y que permitiera independizarse financieramente del apoyo gubernamental, mas no de manera institucional, porque estaban conscientes de que la corresponsabilidad debería privar sobre los distintos enfoques. A pesar de todos los contratiempos, el programa ha continuado gracias a la voluntad de las organizaciones civiles responsables de los módulos.

\section{Funcionamiento de los módulos de atención a migrantes en Tijuana}

En los módulos de atención se recibe y atiende a migrantes deportados desde Estados Unidos dándoles un refrigerio e información sobre los distintos albergues que hay en la ciudad; además se les brinda orientación jurídica sobre sus derechos humanos e información de los lugares adonde pueden acudir en caso de ser violentados, esto mediante una plática informativa y folletos. Al mismo tiempo, se brinda apoyo de teléfono para que se comuniquen con sus familiares y, en algunos casos, se gestionan boletos de autobús para que puedan regresar a sus lugares de origen. A los migrantes que llegan con algún problema de salud, se les canaliza a las instituciones públicas pertinentes y si requieren albergue por algún tiempo, se les deriva y traslada hacia el lugar que ellos escojan. Cuando llegan menores y mujeres se establece comunicación con los organismos que les puedan brindar ayuda inmediata ya que ellos son considerados como población más vulnerable.

En los módulos se desarrollan también monitoreos constantes de las deportaciones para observar si efectivamente se cumple con los acuerdos bilaterales en materia de deportación. Simultáneamente, se utilizan algunas encuestas para observar el cambio en los perfiles migratorios. El proyecto es coordinado por el Departamento de Derechos Humanos de la Coalición, que ha contratado a dos personas para que atiendan de forma permanente a los migrantes deportados. De manera coyuntural, participan voluntarios de la Coalición y estudiantes de servicio social de algunas universidades con las que se han firmado convenios de apoyo y colaboración. También participan miembros de otras organizaciones civiles como Médicos del Mundo, con los que se ha establecido un convenio de colabo- 
ración para atender a los migrantes en temas de salud. La administración de todo el proceso operativo (pagos, gestión de recursos en especie, donaciones, apoyos y todo tipo de trámites ante las instancias gubernamentales) está a cargo del personal de la Coalición.

Los módulos se han convertido en el espacio de referencia tanto para autoridades gubernamentales como para las organizaciones civiles que apoyan a los migrantes, y se han hecho indispensables para la atención de esta población vulnerable.

En palabras de la responsable del programa,

[...] los módulos son los espacios amigables que encuentra el migrante al momento de ser deportado ya que éste llega con niveles graves de incertidumbre y angustia por no saber cuál será su futuro inmediato. Por experiencia, los migrantes tienden a confiar más en el personal de los módulos que en las autoridades de su propio país (entrevista, Esmeralda Siu, 2008).

\section{Un apretado balance de la actividad de los módulos}

El programa de los módulos ha resultado exitoso en sus primeros tres años. Se ha logrado dar visibilidad al fenómeno migratorio local, al difundir la problemática de esta población, así como también la atención de más de 120 mil migrantes en el periodo 2005-2007 en todo el estado y la atención de más de 25 mil por año solamente en las garitas de Tijuana (Informe, 2007). Al mismo tiempo, se ha canalizado hacia los albergues y centros de apoyo a un gran número de migrantes, logrando reducir hasta en $50 \%$ los migrantes que deambulaban por la ciudad, evitando que sean presa de los delincuentes, autoridades corruptas y también que se vean orillados a cometer desmanes por su situación precaria.

En cuanto a la salud de los migrantes, ésta ha mejorado notablemente al contar con atención primaria y detección de enfermedades, y se ha establecido comunicación con las instituciones de salud para darle seguimiento a los que presentan enfermedades infectocontagiosas o viH-Sida. Por otra parte, se han canalizado algunos casos de migrantes a la CNDH para atender violaciones a sus derechos e incumplimientos bilaterales de las condiciones de repatriación. Se ha podido gestionar, también, empleo para los que deciden quedarse a radicar en la ciudad, así como capacitar 
en derechos humanos a un gran número de los que son deportados por las garitas de Tijuana.

La coordinación de los módulos se ha llevado con transparencia y responsabilidad. A la fecha, se han realizado evaluaciones por parte de las instituciones gubernamentales y consultarías externas sobre el uso de los recursos públicos con resultados satisfactorios.

La Coalipro se ha convertido en el interlocutor natural para atender el problema generado por los migrantes. La relación ha sido de mutuo respeto, sin pretender corporativizar a las organizaciones, ni impidiendo que éstas realicen denuncias públicas cuando la acción de gobierno no es la correcta.

Si bien las primeras fases del proyecto han sido bien evaluadas, se pueden observar algunas debilidades de origen: por ejemplo, el proyecto basa la operación de los módulos completamente en recursos públicos, lo cual provoca un estancamiento y su posible clausura a largo plazo; otra debilidad es que la red dedica todos los esfuerzos a los módulos, descuidando otros aspectos de las propias organizaciones; y finalmente, se han descuidado las relaciones con los otros actores de la sociedad civil organizada.

Las tareas de los módulos son sólo una parte de los retos que propone el Comité para desarrollar un programa integral de atención a los migrantes, donde se tiene clara conciencia de que no se debe quedar en un mero asistencialismo, sino lograr incidir en políticas públicas que ayuden a evitar la migración de esta población, incentivando el empleo, la pequeña producción y/o el desarrollo regional. Desgraciadamente, este proyecto integral se ha ido postergando por la incesante actividad de los módulos y la falta de funcionamiento del Comité estatal, que se reúne esporádicamente.

\section{¿Dónde está lo novedoso del proyecto?}

El caso de los módulos de atención a los migrantes deportados desde Estados Unidos a México en Tijuana puede inscribirse en los modelos innovadores de gestión asociada, donde una política pública ya no es aplicada solamente por el sector gubernamental sino es en corresponsabilidad con la sociedad civil.

La organización responsable de los módulos sigue manteniendo su autonomía y responde críticamente cuando considera que las políticas gu- 
bernamentales no están funcionando o se están desviando de su cometido. Sin embargo, esa actitud poco a poco ha ido disminuyendo por las fricciones que le generan con las autoridades que fungen como socias.

Desde otra perspectiva, los módulos de atención al migrante en Tijuana pueden ser considerados como exitosos en cuanto a la incidencia en la política pública local o por la participación de las organizaciones civiles agrupadas en red en todas las fases de la política pública, desde la construcción del problema público, pasando por la incorporación del problema en la agenda, hasta la formulación de la política pública, su implementación, el seguimiento y la evaluación. En el repertorio de oportunidades, la red Coalipro hizo uso de su capacidad de diseño en políticas públicas, ya que las organizaciones integrantes tienen varios años atendiendo el problema con pocos apoyos gubernamentales y con un amplio conocimiento de la problemática; asimismo, la Coalipro se presenta como un interlocutor serio no sólo por sus acciones contestatarias y sus propuestas alternativas, sino también por su actitud colaboracionista.

Cabe agregar que la red Coalipro tuvo la capacidad organizativa suficiente, que terminó por eliminar los temores del sector gubernamental en cuanto a dejar en manos de la sociedad civil acciones de política pública con recursos públicos; esas fortalezas fueron valorizadas por los demás integrantes del Comité Estatal, de tal forma que le otorgaron la dirección de las acciones de los módulos.

Respecto de las estrategias implementadas por la Coalipro, puede afirmarse que el proyecto ha sido exitoso también por las alianzas que estableció con otras redes y organismos civiles, como la Red Pastoral Social de Mexicali y las universidades Iberoamericana y Autónoma de Baja California, así como con El Colegio de la Frontera Norte. Hay que agregar que el posicionamiento obtenido, el cual ha servido para influir en una política migratoria de Estado, se debe a su constante participación en comités y consejos relacionados con esta problemática, a las denuncias constantes sobre violaciones a los derechos humanos de los migrantes, a la presentación de propuestas alternativas de políticas públicas, al uso constante de los medios de comunicación locales y nacionales y a la gran capacidad para que la población tome conciencia sobre este problema. 


\section{Consideraciones finales}

Los obstáculos para desarrollar procesos de gestión innovadores en el país, especialmente para llevar a cabo programas de cogestión, gestión asociada u otro tipo, son múltiples, y van desde los intereses partidistas, la miopía de los gobernantes, la ignorancia e incapacidad para vislumbrar nuevas formas de democracia desde abajo, hasta las resistencias por parte de las organizaciones civiles a participar por temor a ser captadas y utilizadas para fines políticos partidistas. Lo anterior es consecuencia de la falta de una cultura de gestión pública tanto de los gobiernos locales como de las organizaciones civiles. Por eso, en el caso de la Oficina de Atención a Migrantes en Mexicali, la mayor resistencia para la instalación del módulo provenía del gobierno federal, porque no concebía la posibilidad de que una organización civil pudiera desarrollar un programa público y menos en terrenos donde se desarrollan acciones que se alejan mucho de la transparencia. Afortunadamente, otros factores, como el prestigio y la seriedad del trabajo de las oc y las relaciones de los encargados en distintos niveles, influyeron para que las autoridades terminaran por aceptar el programa.

En nuestro caso, no se pudieron cumplir algunas de las condiciones que Clemente (2000) y Ramos y Reyes (2005) apuntaban como indispensables para lograr una gestión asociada, pues por un lado el gobierno no permitió la autonomía de los actores sociales y los seguía concibiendo con la visión de subordinación, es decir, no mostró voluntad de buscar la cogestión, mucho menos favorecer el fortalecimiento de sus capacidades; por otro, las organizaciones civiles tampoco mostraron mucha disposición para desarrollar un programa de corresponsabilidad donde el gobierno tuviera injerencia; además la actitud de las organizaciones civiles tampoco propiciaba un ambiente de transparencia.

Como bien lo han mencionado Ramos y Reyes (2005), muchos de los intentos innovadores no siempre han prosperado en nuestro país y las oc terminan por no incidir verdaderamente en las políticas públicas, esto porque los gobiernos locales no han establecido una visión de gestión asociada; o bien porque cuando han incorporado la visión de la nueva gestión pública, lo han hecho de una manera distorsionada, incorporando solamente las nuevas técnicas de la administración pública y no la filosofía que 
le da sustento, lo que ha impedido que la sociedad civil organizada pueda incidir en las políticas de desarrollo social (Ramos y Reyes, 2005).

La Oficina de Atención al Migrante en Mexicali sigue funcionando de manera regular, los conflictos con las organizaciones gubernamentales han disminuido y se mantiene una relación de mutuo respeto que poco a poco va disipando los temores en ambos lados para abrir paso a procesos incipientes de colaboración y corresponsabilidad. A pesar de que la oficina no recibe recursos del gobierno en forma directa que pudieran potenciar sus actividades, ahora es vista por el gobierno como un posible aliado estratégico para atender a la población migrante deportada en Mexicali.

En el caso de los módulos de atención a migrantes en Tijuana, a pesar de todos los contratiempos, el programa continúa gracias a la voluntad y los esfuerzos de las organizaciones civiles, y resulta un proyecto prometedor para la construcción de una nueva democracia desde lo local. Aquí sí se cumplieron desde el principio las condiciones elementales para desarrollar un proceso de gestión asociada, es decir, la autonomía de las partes, la voluntad del gobierno por trabajar bajo el esquema de cogestión, la transparencia en el uso de los recursos y la apertura a una nueva cultura de gestión. Lo anterior a pesar de que el proceso no ha estado exento de intentos por corporativizar a la red de organizaciones civiles. Se observa que tanto el gobierno como la sociedad civil están aprendiendo a desarrollar sus responsabilidades sin la tradicional "cobra de factura política", lo cual puede ser considerado como un gran avance.

El programa de los módulos de atención al migrante en Tijuana ha resultado interesante al gobierno federal, de tal manera que en marzo de 2008 inició un programa piloto para la atención a migrantes repatriados al que se denominó Programa de Repatriación Humana (Salinas, 2008).

A pesar de todos los obstáculos, los dos ejemplos mostrados en esta investigación pueden ser considerados innovadores en el contexto mexicano porque rompen con los esquemas tradicionales de gestión pública y constituyen el germen de una gobernanza local.

\section{Bibliografía}

Cabrero Mendoza, Enrique (2003), "La agenda de políticas públicas en el ámbito municipal: una visión introductoria”, en Enrique Cabrero, Políticas públicas municipales, una agenda en construcción, México, Miguel Ángel Purrúa. 
Canto Chac, Manuel (2002), "Introducción a las políticas publicas”, en Manuel Canto y Castro Soto (coords.), Participación ciudadana y políticas públicas en el municipio, México, MCD.

Clemente, Adriana (2000), "El papel de las ONG en el desarrollo local", Sintesis. Política y Desarrollo Local en Iberoamérica, Madrid, núms. 33-34, s/f, pp. 111129.

Fernández Santos, Yolanda, José Miguel Fernández y Alicia Rodríguez Pérez Fernández (2008), "Modernización de la gestión pública. Necesidad, incidencias, límites y críticas”, Pecunia, Revista de la Facultad de Ciencias Económicas y Empresariales de la Universidad de León, España, núm. 6, pp. 75105.

Guillén López, Tonatiuh, Liliana González Pantoja y Pablo Rojo Calzada (coords.), Gobiernos de proximidad. La capacidad y el ingenio de la gestión local mexicana, México, CIDE, Segob, El Colef.

Gómez Castellanos, Rodolfo y Patricia Moctezuma (1999), "Las políticas públicas", en Societas, Revista del Colegio Estatal de Sociólogos de Baja California, Mexicali, año 3 , núm. 11.

Colipro (2007), Informe de migrantes atendidos y servicios otorgados por la Coalición Pro Defensa del Migrante, A.C. a través del Módulo de Atención a Migrantes Deportados Puerta México en Tijuana, Baja California, Departamento de Derechos Humanos, enero-agosto de 2007, 5 pp., mimeo.

—, Segundo Informe sobre el Monitoreo por el Frente Amplio Pro Defensa del Migrante, 1997, 4 pp., mimeo.

Vargas Hernández, José G. (2005), "Neocolonialismo, resistencia, crisis y transformación del Estado", Revista Internacional de Ciencias Sociales y Humanidades, sociotam, Universidad Autónoma de Tamaulipas, Ciudad Victoria, México, año/vol. XV, número 002, julio-diciembre, pp. 155-188.

Olvera, Alberto J. (2001), "Sociedad civil, gobernabilidad democrática, espacios públicos y democratización: los contornos de un proyecto en México", Cuadernos de la Sociedad Civil, Universidad Veracruzana, núm. 1.

Poggiese, Héctor y María del C. Francioni (1993), "Escenarios de gestión asociada y nuevas fronteras entre el Estado y la sociedad”, en Héctor Poggiese y María del C. Francioni, Escenarios de gestión asociada y nuevas fronteras entre el Estado y la sociedad, Buenos Aires, Argentina, Facultad Latinoamericana de Ciencias Sociales, 1993.

Ramos, José María y Marcela Reyes (2005), “Gobiernos locales y participación ciudadana: hacia un enfoque de gestión estratégica asociada", Espiral. Estudios sobre Estado y Sociedad, vol. XII, núm. 34, septiembre/diciembre de 2005, pp. 39-66. 
Reyes, Marcela (2008), Organizaciones civiles, gestión asociada y desarrollo local desde un enfoque glocal, tesis de doctorado en Estudios del Desarrollo Global, UABC, México.

Revista Migrantes, Revista de información y análisis de los Misioneros de San Carlos/ Scalabrininos, año XI, núm. 3, abril-julio, Tijuana, Baja California, 2005.

Salinas, Daniel (2008), "Arranca el programa de repatriación humana”, Frontera. info 31 de marzo del 2008. Tijuana, disponible en http://www.frontera.info/ EdicionEnLinea/Notas/Noticias/31032008/295693.aspx, consultado el 31 de marzo del 2008.

Sánchez, Lugo Lizeth (2000), "Las organizaciones civiles frente a la problemática migratoria. Una esperanza para los migrantes", entrevista a Blanca Villaseñor, en Paralelo 28, publicación bimestral para los bajacalifornianos, núm. 1, 1 de enero de 2000, pp.1-2.

Wagner, Fritz (2007), "Buena gobernanza, gobernabilidad y políticas públicas dirigidas hacia la atención de los retos de salud", Dirección General de Promoción a la Salud, en las megaciudades y grandes aglomeraciones urbanas en la región de la ops/oms, en Primera reunión del Foro Regional de Salud Urbana de la Organización Panamericana de la Salud, 27 al 29 de noviembre de 2007, México. Disponible en http://www.dgps.salud.gob.mx/interior/ salud_urbana.html

\section{Entrevistas:}

Mónica Oropeza (2009), Albergue del Desierto.

Esmeralda Siu (2008), coordinadora del Departamento de Derechos Humanos de la Coalición Pro Defensa del Migrante A.C.

Artículo recibido en junio de 2009

Segunda versión recibida en noviembre de 2009

Artículo aprobado en diciembre de 2009 